\title{
Vision and touch: Multiple or multisensory representations of objects?
}

\author{
Simon Lacey, Christine Campbell $\uparrow$, K Sathian§ \\ Department of Neurology, School of Medicine, Emory University, Atlanta, GA 30322, USA; \\ - Anglia Ruskin University, Cambridge CB1 1PT, UK; § also Atlanta VA Rehabilitation R\&D Center \\ of Excellence, Decatur, GA 30033, USA; e-mail: slacey@emory.edu \\ Received 9 October 2006, in revised form 4 April 2007
}

\begin{abstract}
The relationship between visually and haptically derived representations of objects is an important question in multisensory processing and, increasingly, in mental representation. We review evidence for the format and properties of these representations, and address possible theoretical models. We explore the relevance of visual imagery processes and highlight areas for further research, including the neglected question of asymmetric performance in the visuo-haptic cross-modal memory paradigm. We conclude that the weight of evidence suggests the existence of a multisensory representation, spatial in format, and flexibly accessible by both bottom - up and top-down inputs, although efficient comparison between modality-specific representations cannot entirely be ruled out.
\end{abstract}

\section{Introduction}

The relationship between visual perception and representation, when representations are derived from a single perceptual modality, has been extensively debated (see Pylyshyn 2003; Kosslyn et al 2006). Discussion has also extended to representations derived from multiple modalities (Calvert et al 2004). Here, we review the relationship between haptic and visual representation of objects, and explore whether a single, multisensory representation or multiple, modality-specific representations better fit the existing evidence. We focus particularly on cross-modal memory research, because of our interest in the nature of the representation: these investigations employ a study-test task in which vision and touch are separated by a delay. Success on this task depends on encoding, storing, and retrieving a representation; thus, cross-modal memory is a useful tool with which to explore the nature of the representation(s). However, we also draw on other approaches, such as neuroimaging and priming, and studies with the blind.

\section{Format of visuo - haptic object representation}

At the outset, we need to distinguish between representational format and content (Kosslyn et al 2006): the format is the code in which representation occurs whilst the content is the information conveyed. This distinction is especially important for representations whose content can be derived from multiple modalities and which therefore have several potential formats.

\subsection{Dual-code and visual imagery accounts}

Johnson et al (1989) proposed a dual-code account of cross-modal object representation, arguing that haptic input activates visual representations for both familiar and unfamiliar objects, but that this activation spreads to a verbal representation for familiar objects since these can be named. Although the performance of children instructed to use either visual or verbal strategies correlated with their visuo-spatial and verbal abilities (Johnson et al 1989), only familiar nameable shapes were used and thus the role of familiarity was not rigorously tested. Since cross-modal memory has been demonstrated in preverbal infants and several animal species, it is unlikely that language acquisition or verbal strategies are fundamental to performance (Rose 1994). However, verbal processes may 
play a facilitatory role: unfamiliar objects may not be nameable but they can be described (see the procedure in Easton et al 1997a). Lacey and Campbell (2006a) found that a verbal secondary task disrupted encoding of unfamiliar, but not familiar, objects and interpreted this as interference with a strategy of covert verbal description. Coding the spatial relationship of different features within the same object can be dissociated from coding the relationship between the spatial locations of different objects (Cooper and Humphreys 2000) and recent work shows that verbal descriptions of 'betweenobject' relationships are functionally equivalent to perceptually derived representations of these relationships (Avraamides et al 2004). The multisensory and 'within-object' implications of these findings have not been fully explored, although the piecemeal construction of representations of imaginary objects from verbal instructions conveying spatial (directional) information engages the dorsal visual pathway (Mellet et al 1996). We also note that all objects follow an unfamiliar - familiar trajectory: thus the question arises whether representations change over this trajectory and how this relates to individual differences.

A visual imagery account is more parsimonious than a dual-code account because visual information is available for both familiar and unfamiliar objects, and thus only a single code is required. This account suggests a relationship between cross-modal memory performance and visual imagery ability. Visuo-spatial abilities of children are indeed correlated with cross-modal memory performance for both familiar (Johnson et al 1989) and unfamiliar objects (Stoltz-Loike and Bornstein 1987); and 'good' imagers, as measured by the Vividness of Visual Imagery Questionnaire (VVIQ) (Marks 1973), are better at cross-modal memory than 'poor' imagers (Cairns and Coll 1977). However, there are problems with this approach. A single test of imagery ability is probably inappropriate because visual imagery is a multi-component process including image generation, maintenance, inspection, and transformation, and individuals may vary on any or all of these components (Kosslyn et al 1994). The VVIQ potentially measures only a subjective aspect of image quality or experience, rather than the underlying imagery processes and abilities themselves (Dean and Morris 2003).

'Good' imagers may perform well, not because they are more proficient at imagery, but because they make better use of relevant information (Baddeley and Andrade 2000). Furthermore, different imagery abilities may contribute differentially to crossmodal memory and representation: spatial recall predicts cross-modal performance whilst mental rotation does not (Stoltz-Loike and Bornstein 1987). These studies raise the question of how far visual imagery processes can be mapped onto accounts of cross-modal memory processes. For instance, is the ability to generate images more important for touch than for vision and for unfamiliar objects than for familiar ones?

An important question is how haptic input could be translated into a visual image, given that the sensory impressions are so different (Rose 1994) and that vision and touch use different metrics and geometries (Kappers and Koenderink 1999). However, there is substantial neuroimaging evidence showing that vision and touch are intimately connected even if there is no direct, one-to-one mapping (see Amedi et al 2005; Sathian 2005 for reviews). Cerebral cortical areas previously regarded as exclusively visual are activated by haptic/tactile input in a task- and stimulus-specific manner. Visual cortical areas are activated during haptic/tactile perception of grating orientation (Sathian et al 1997), motion (Blake et al 2004), and form (Amedi et al 2001; Prather et al 2004). Significantly, inter-individual variations in the strength of haptic shape-selective activation of the lateral occipital complex (LOC), a visual shape-selective area, are predicted by inter-individual variations in imagery scores (Zhang et al 2004). It is worth noting that this correlation was only found for the right, and not the left, LOC even though haptic exploration was with the right hand (Zhang et al 2004) - this raises the possibility of hemispheric specialization of the imagery-related processes. 


\subsection{Modality-independent representations}

Nonetheless, do these activations, systematic and organized as they are, really reflect a specifically visual representation? A third approach argues that vision and touch encode object properties common to both modalities into a single, modality-independent representation. Since spatial information is available for all objects and can be perceived by both vision and touch, such a representation is likely spatial. Lacey and Campbell (2006b) showed that spatial interference disrupted both encoding and retrieval, regardless of whether the interference task was presented visually or haptically. Non-spatial interference had no effect, again regardless of modality. It is notable that spatial interference eliminated asymmetric performance (see below) and affected both encoding and retrieval of representations (Lacey and Campbell 2006b). When interference disrupts encoding, it is difficult to say whether the effect reflects the format of the representation or its content: it might simply disrupt perception of the information to be encoded. Retrieval, by contrast, is a process of recalling the representation from store and does not involve perception of the object. Thus, when interference disrupts retrieval, especially if the encoding and retrieval modalities are different, it is more likely that this reflects a property of the retrieved representation than a property of the object. This logic suggests that the interference effect is related to a common format rather than a common content. A spatial-representation model of cross-modal memory is ostensibly the most parsimonious account, since spatial information is available for all objects and can be perceived by both vision and touch.

Whether there is a single multisensory spatial representation or separately derived visual and haptic spatial representations remains uncertain, but has important implications for the development of theoretical models of cross-modal memory. A single representation implies a translation model, whereas separate representations imply a comparison model. A recent neuroimaging study shows that category selectivity within human inferotemporal cortex is relatively independent of whether the input modality is visual or haptic (Pietrini et al 2004), suggesting a multisensory representation for objects. A patient with visual agnosia (inability to recognize objects), following a lesion of the left occipito-temporal cortex also turned out to have tactile agnosia, although somatosensory cortex and sensation were intact (Feinberg et al 1986). Another patient who developed bilateral lesions of the LOC was impaired at learning unfamiliar objects using either touch or vision (James et al 2006). These studies suggest that a shared representation is disrupted and thus also favor a multisensory representation. However, Reed et al (1996) report a patient, EC, with tactile but not visual agnosia following a lesion of ventrolateral somatosensory cortex. This suggests that there may be a unisensory haptic representation in somatosensory cortex, in addition to a multisensory representation in visual cortex.

\section{How can different representational formats be distinguished experimentally?}

Much of the empirical evidence for modality-independent representation derives from priming studies. Repetition priming makes inferences about the nature of representations based on responses to a probe stimulus delivered after an initial priming stimulus. Thus, from the finding that cross-modal priming is equal to within-modal priming, it is inferred that the representations of both prime and probe are common to vision and touch, and that these representations are primarily structural (Easton et al 1997a, 1997b; Reales and Ballesteros 1999). Converging on this conclusion, an fMRI study showed similar within- and cross-modal priming effects in the LOC when unfamiliar objects were presented visually during scanning following either visual or haptic pre-exposure, suggesting that vision and touch share geometric information (James et al 2002). However, a potential problem is that, when more than one modality is involved, priming effects cannot necessarily distinguish between competing candidates for the representational format. 
For example, the efficient translation of (a) haptic input into a visual representation; or (b) haptic and visual input into another, modality-independent representation; or (c) the efficient comparison of separate modality-specific visual and haptic representations, might all result in equal within- and cross-modal priming. Interference techniques may be a stronger test of cross-modal representation than the priming paradigm - these techniques have been successful in distinguishing visual, spatial, and verbal elements of working memory (eg Baddeley et al 1975; McConnell and Quinn 2000). Interference tasks in the cross-modal paradigm were originally studied with the tasks placed in the study - test interval (Millar 1972; Garvill and Molander 1977); however, this placement is unlikely to reveal anything about representational format (Quinn and McConnell 2006). As discussed above, interference tasks during encoding and retrieval have been helpful in establishing the spatial format of cross-modally accessible representation(s) (Lacey and Campbell 2006a, 2006b). Further refinement of the interference paradigm may be helpful to explore the nature of multisensory representation in more detail.

\section{Imagery processes and individual differences}

If haptic and visual perception engage modality-independent imagery systems, we might expect to see similarities between visually and haptically derived imagery processes. In visual imagery, scanning times increase with the spatial distance to be inspected in the image, implying that visual images preserve metric spatial information (Kosslyn et al 1978). The same effect is observed in scanning haptically derived representations, implying that these are also spatial and that similar, if not identical, imagery processes operate in both modalities (Röder and Rösler 1998). Studies of mental rotation of visual stimuli show that the time taken to judge whether two objects are the same or mirror-images increase linearly with increasing angular disparity between the stimuli (Shepard and Metzler 1971). This also holds true for haptic and tactile stimuli (Dellantonio and Spagnolo 1990; Prather and Sathian 2002). Mental rotation of tactile stimuli activates the left anterior intraparietal sulcus (Prather et al 2004), an area that is also active during mental rotation of visual stimuli (Alivisatos and Petrides 1997), further supporting the idea that similar spatial imagery processes operate on both visually and haptically derived representations.

Interestingly, these similarities between visually and haptically derived representations are observed in both the early- and late-blind as well as the sighted (Röder and Rösler 1998). The blind may be slower than the sighted at haptic mental rotation (Marmor and Zaback 1976), but Hollins (1986) suggests that, after controlling for greater variability in the use of frames of reference by the sighted, this difference is more apparent than real. In addition, both the blind and sighted show activation in the LOC, while generating mental images of shape derived from haptic and visual experience, respectively (De Volder et al 2001). The LOC is also active when visual and haptic object properties are retrieved from memory (Newman et al 2005). These studies indicate that common processes are engaged when generating, retrieving, and manipulating representations derived from both vision and touch.

Recent work by Kozhevnikov and colleagues (Kozhevnikov et al 2005; Blajenkova et al 2006) has shown that visual imagery is of two distinct types. 'Object imagery' refers to images that are more pictorial and detailed in terms of shape, color, brightness, etc, whilst 'spatial imagery' refers to images that are more concerned with the spatial relations of objects and object parts and hence may facilitate spatial transformations. Thus, an interesting question is whether these different types of imagery can be related to the input modality: for example, are spatial imagers better at haptically deriving representations than object imagers, and would they therefore perform better on the cross-modal memory task? 
Whilst some researchers have argued that visual imagery cannot mediate haptic perception on the basis that congenitally blind individuals can still recognize objects (eg Pietrini et al 2004; Goyal et al 2006), this does not rule out a role for visual imagery in the normally sighted. Moreover, spatial imagery activates a common pathway in both sighted and blind (Vanlierde et al 2003), so that spatial representation offers a way of reconciling the imagery and modality-independent accounts.

\section{General properties of visuo - haptic representation}

The more general properties of visuo - haptic representation are less well-defined. One general property is viewpoint-dependence. Both visual and haptic object recognition appear to be viewpoint-dependent since recognition performance was reduced when objects were rotated $180^{\circ}$ away from the learned view - the 'front' surface for vision and the 'back' surface for touch (Newell et al 2001). Interestingly, cross-modal recognition also showed an effect of viewpoint but recognition of rotated objects actually improved. This was attributable to vision and touch favoring opposite surfaces: when the 'front/visual' view of the object was rotated, it became the 'back/haptic' and viceversa (Newell et al 2001). Although this preference may reflect no more than the biomechanics of the hand, it may also imply that visual and haptic spatial maps are initially separate and only combine later in the service of object recognition.

It is unclear whether the bimodally accessible representation(s) is (are) globally based, encoding representations of whole objects, or locally based, encoding the spatial organization of the local parts. This is important because local features are more salient than global features in haptic perception as compared to visual perception (Lakatos and Marks 1999; but see also Heller and Clyburn 1993). In visual representation, this has been investigated by examining the rate of mental rotation. Since representations of possible and impossible objects were rotated at roughly the same rates, Dror et al (1997) argued that visual representations were locally based because encoding the global shape of impossible objects would quickly reveal their structural impossibility and slow their rotation compared to possible objects, whereas local parts could be rotated successfully in isolation. The implications for multisensory processing are unexplored but one could expect that, for example, similar objects would evoke representations distinguished predominantly in local aspects owing to shared global shape, whereas dissimilar objects would evoke representations that could differ in both global and local aspects.

\section{Asymmetric cross-modal memory performance}

Cross-modal memory performance is generally significantly better when visual encoding is followed by haptic retrieval than the reverse (Streri and Molina 1994; Lacey and Campbell 2006a, 2006b). Although this asymmetric performance is a consistent aspect of cross-modal memory studies, it has not received much attention. If cross-modal memory relied on a single representational format, based on properties that can be represented equally well by different modalities, then performance should be similar in both visual - haptic and haptic - visual conditions (Freides 1974; Streri and Molina 1994). Asymmetric performance would thus seem to argue against a single representation, but other explanations need to be ruled out. For instance, modality-independent information may not be encoded equally well in vision and touch because of competition from more salient modality-specific information. Thus, visual imagery instructions have been shown to reverse the haptic salience of properties from (in descending order of salience) hardness - texture-shape to shape-texture-hardness/size (Klatzky et al 1987). One interpretation of this is that the imagery-biased instructions made it difficult to encode hardness and texture information that is normally more salient haptically and thus brought shape information, common to both modalities, to the fore. A principled manipulation of object properties is required to examine these possibilities. 
A simpler explanation might be that haptic working memory capacity appears to be more limited and variable (Bliss and Hämäläinen 2005) and thus more error-prone than that of visual working memory. Another is that haptic representations may simply decay faster than visual representations. Certainly, the decay function in haptic memory, rather than being progressive, appears to occur entirely in a band of 15-30 seconds post-stimulus (Kiphart et al 1992). This might suggest that performance would be worse in the haptic-visual condition of the cross-modal memory task because haptically encoded representations have substantially decayed by the time that visual retrieval is required. But several cross-modal memory studies show that increasing the delay between encoding and retrieval up to 30 seconds did not affect haptic-visual performance more than visual - haptic performance (Garvill and Molander 1973; Woods et al 2004) and so it seems that the explanation will be more complicated than a simple function of haptic memory properties alone.

Interestingly, asymmetry is also observed in the infant-developmental literature. These studies attribute asymmetric performance to constraints imposed by different stages of motor development (Streri and Molina 1994). But since asymmetry persists beyond early development into more mature motor systems (Easton et al 1997a, 1997b; Bushnell and Baxt 1999; Lacey and Campbell 2006a, 2006b), it is likely that other representationally important factors are involved. The asymmetry observed in studies of explicit memory does not seem to apply to implicit memory: cross-modal priming is apparently symmetric (Easton et al 1997a, 1997b; Reales and Ballesteros 1999), although influences of verbal encoding cannot entirely be ruled out in these studies.

\section{Conclusions}

We have reviewed what is currently known about visuo-haptic object representation and suggested new research questions about the role of verbal strategies, imagery processes, object familiarity, general image properties, and performance asymmetries. The most important goal, however, will be the development of a theoretical model, particularly in relation to understanding individual differences. We conclude that the current weight of evidence suggests the existence of a multisensory representation, spatial in format, and flexibly accessible via both bottom-up and top-down inputs, although the alternative of efficient comparison between modality-specific representations cannot be ruled out with certainty, and additional, unisensory representations may also be present. Connectivity patterns are likely to be helpful in resolving these issues. Although such patterns have not been as extensively explored in the visuohaptic domain as in the audio-visual domain (eg Schroeder et al 2003), an effective connectivity study revealed both bottom-up and top-down paths in a network of visuo-haptically shape-selective areas, including foci in the postcentral sulcus, the intraparietal sulcus, and the LOC (Peltier et al 2007). Finally, although we can readily appreciate the adaptive advantage of cross-modal memory, what is its evolutionary significance? Is the ability to represent objects and potential objects by vision and touch the cognitive advance that enabled the development of advanced stone-tool industries (eg Schlanger 1996) and thus propelled early hominids ahead of other primates? These questions and possibilities make visuo - haptic representation, and multisensory processing in general, the most compelling of problems.

Acknowledgment. Support to KS from the National Eye Institute at the NIH, the National Science Foundation, and the Veterans Administration is gratefully acknowledged. 


\section{References}

Alivisatos B, Petrides M, 1997 "Functional activation of the human brain during mental rotation" Neuropsychologia $36111-118$

Amedi A, Kriegstein K von, Atteveldt N M van, Beauchamp M S, Naumer M J, 2005 "Functional imaging of human cross-modal identification and object recognition" Experimental Brain Research $166559-571$

Amedi A, Malach R, Hendler T, Peled S, Zohary E, 2001 "Visuo-haptic object-related activation in the ventral visual pathway" Nature Neuroscience 4 324-330

Avraamides M N, Loomis J M, Klatzky R L, Golledge R G, 2004 "Functional equivalence of spatial representations derived from vision and language: evidence from allocentric judgments" Journal of Experimental Psychology: Learning, Memory, and Cognition 30801 - 814

Baddeley A D, Andrade J, 2000 "Working memory and the vividness of imagery" Journal of Experimental Psychology: General 129 126-145

Baddeley A D, Grant S, Wight E, Thomson M, 1975 "Imagery and visual working memory", in Attention \& Performance V Eds P M A Rabbitt, S Dornic (London: Academic Press) pp 205-217

Blajenkova O, Kozhevnikov M, Motes M A, 2006 "Object-spatial imagery: a new self-report imagery questionnaire" Applied Cognitive Psychology $20239-263$

Blake R, Sobel K V, James T W, 2004 "Neural synergy between kinetic vision and touch" Psychological Science $15397-402$

Bliss I, Hämäläinen H, 2005 "Different working memory capacity in normal young adults for visual and tactile letter recognition task" Scandinavian Journal of Psychology 46 247-251

Bushnell E W, Baxt C, 1999 "Children's haptic and cross-modal recognition with familiar and unfamiliar objects" Journal of Experimental Psychology: Human Perception and Performance $251867-1881$

Cairns E, Coll P, 1977 "The role of visual imagery in visual, tactual and cross-modal matching" British Journal of Psychology $68213-214$

Calvert G, Spence C, Stein B (Eds), 2004 The Handbook of Multisensory Processes (Cambridge, MA: MIT Press)

Cooper A C G, Humphreys G W, 2000 "Coding space within but not between objects: evidence from Balint's syndrome" Neuropsychologia $38723-733$

Dean G, Morris P E, 2003 "The relationship between self-reports of imagery and spatial ability" British Journal of Psychology $94245-273$

Dellantonio A, Spagnolo F, 1990 "Mental rotation of tactual stimuli" Acta Psychologica 73 245-257

De Volder A G, Toyama H, Kimura Y, Kiyosawa M, Nakano H, Vanlierde A, Wanet-Defalque M-C, Mishina M, Oda K, Ishiwata K, Senda M, 2001 "Auditory triggered mental imagery of shape involves visual association areas in early blind humans" NeuroImage $14129-139$

Dror I E, Ivey C, Rogus C, 1997 "Visual mental rotation of possible and impossible objects" Psychonomic Bulletin \& Review $4242-247$

Easton R D, Greene A J, Srinivas K, 1997a "Transfer between vision and haptics: memory for 2-D patterns and 3-D objects" Psychonomic Bulletin \& Review 4 403-410

Easton R D, Srinivas K, Greene A J, 1997b "Do vision and haptics share common representations? Implicit and explicit memory within and between modalities" Journal of Experimental Psychology: Learning, Memory, and Cognition 23 153-163

Feinberg T E, Rothi L J, Heilman K M, 1986 "Multimodal agnosia after unilateral left hemisphere lesion" Neurology $36864-867$

Freides D, 1974 "Human information processing and sensory modality: cross-modal functions, information complexity, memory and deficit" Psychological Bulletin 8 284-310

Garvill J, Molander B, 1973 "Effects of standard modality, comparison modality and retention interval on matching of form" Scandinavian Journal of Psychology 14 203-206

Garvill J, Molander B, 1977 "Effects of interference on intra-modal and cross-modal matching of form", Umeå Psychological Reports, No 124

Goyal M S, Hansen P J, Blakemore C B, 2006 "Tactile perception recruits functionally related visual areas in the late-blind" NeuroReport $171381-1384$

Heller M A, Clyburn S, 1993 "Global versus local processing in haptic perception of form" Bulletin of the Psychonomic Society $31574-576$

Hollins M, 1986 "Haptic mental rotation: more consistent in blind subjects?" Journal of Visual Impairment and Blindness 80 950-952

James T W, Humphrey G K, Gati J S, Servos P, Menon R S, Goodale M A, 2002 "Haptic study of three-dimensional objects activates extrastriate visual areas" Neuropsychologia 40 1706-1714 
James T W, James K H, Humphrey G K, Goodale M A, 2006 "Do visual and tactile object representations share the same neural substrate?", in Touch and Blindness: Psychology and Neuroscience Eds M A Heller, S Ballesteros (Mahwah, NJ: Lawrence Erlbaum Associates) pp $139-155$

Johnson C L, Paivio A U, Clark J M, 1989 "Spatial and verbal abilities in children's cross-modal recognition: a dual-coding approach" Canadian Journal of Psychology 43 397-412

Kappers A M L, Koenderink J J, 1999 "Haptic perception of spatial relations" Perception 28 $781-795$

Kiphart M J, Hughes J L, Simmons J P, Cross H A, 1992 "Short-term haptic memory for complex objects" Bulletin of the Psychonomic Society $30212-214$

Klatzky R L, Lederman S, Reed C, 1987 "There's more to touch than meets the eye: the salience of object attributes for haptics with and without vision" Journal of Experimental Psychology: General $116356-369$

Kosslyn S M, Ball T M, Reiser B J, 1978 "Visual images preserve metric spatial information: evidence from studies of image scanning" Journal of Experimental Psychology: Human Perception and Performance 4 47-60

Kosslyn S M, Brunn J, Cave K R, Wallach R W, 1984 "Individual differences in mental imagery ability" Cognition $18195-243$

Kosslyn S M, Thompson W L, Ganis G, 2006 The Case for Mental Imagery (Oxford: Oxford University Press)

Kozhevnikov M, Kosslyn S M, Shephard J, 2005 "Spatial versus object visualizers: a new characterization of visual cognitive style" Memory \& Cognition 33 710-726

Lacey S, Campbell C, 2006a "Mental representation in visual/haptic cross-modal memory: evidence from interference effects" Quarterly Journal of Experimental Psychology 59361 - 376

Lacey S, Campbell C, 2006b Object Representation in Visual/Haptic Cross-Modal Memory 7th International Multisensory Research Forum (abstract)

Lakatos S, Marks L E, 1999 "Haptic form perception: relative salience of local and global features" Perception \& Psychophysics $61895-908$

Marks D F, 1973 "Visual imagery differences in the recall of pictures" British Journal of Psychology $6417-24$

Marmor G S, Zaback L A, 1976 "Mental rotation by the blind: does mental rotation depend on visual imagery?" Journal of Experimental Psychology: Human Perception and Performance 2 $515-521$

McConnell J, Quinn J G, 2000 "Interference in visual working memory" Quarterly Journal of Experimental Psychology A $5353-67$

Mellet E, Tzourio N, Crivello F, Joliot M, Denis M, Mazoyer B, 1996 "Functional anatomy of spatial imagery generated from verbal instructions" Journal of Neuroscience 16 6504-6512

Millar S, 1972 "Effects of interpolated tasks on latency and accuracy of intermodal and crossmodal shape recognition by children" Journal of Experimental Psychology 96 170 - 175

Newell F N, Ernst M O, Tjan B S, Bülthoff H H, 2001 "Viewpoint dependence in visual and haptic object recognition" Psychological Science 12 37-42

Newman S D, Klatzky R L, Lederman S J, Just M A, 2005 "Imagining material versus geometric properties of objects: an fMRI study" Cognitive Brain Research 23235 - 246

Peltier S, Stilla R, Mariola E, LaConte S, Hu X, Sathian K, 2007 "Activity and effective connectivity of parietal and occipital cortical regions during haptic shape perception" Neuropsychologia $45476-483$

Pietrini P, Furey M L, Ricciardi E, Gobbini M I, Wu W-H C, Cohen L, Guazzelli M, Haxby J V, 2004 "Beyond sensory images: object-based representation in the human ventral pathway" Proceedings of the National Academy of Sciences of the USA $1015658-5663$

Prather S C, Sathian K, 2002 "Mental rotation of tactile stimuli" Cognitive Brain Research 14 $91-98$

Prather S C, Votaw J R, Sathian K, 2004 "Task-specific recruitment of dorsal and ventral visual areas during tactile perception" Neuropsychologia 421079 - 1087

Pylyshyn Z W, 2003 Seeing and Visualizing: It's Not What You Think (Cambridge, MA: MIT Press)

Quinn J G, McConnell J, 2006 "The interval for interference in conscious visual imagery" Memory $14241-252$

Reales J M, Ballesteros S, 1999 "Implicit and explicit memory for visual and haptic objects: crossmodal priming depends on structural descriptions" Journal of Experimental Psychology: Learning, Memory, and Cognition $25644-663$

Reed C L, Caselli R J, Farah M J, 1996 "Tactile agnosia: underlying impairment and implications for normal tactile object recognition" Brain $119875-888$ 
Röder B, Rösler F, 1998 "Visual input does not facilitate the scanning of spatial images" Journal of Mental Imagery $22165-181$

Rose S A, 1994 "From hand to eye: findings and issues in infant cross-modal transfer", in The Development of Intersensory Perception: Comparative Perspectives Eds D J Lewkowicz, R Lickliter (Hove, Sussex: Lawrence Erlbaum Associates) pp 265-284

Sathian K, 2005 "Visual cortical activity during tactile perception in the sighted and the visually deprived" Development Psychobiology 46 279-286

Sathian K, Zangaladze A, Hoffman J M, Grafton S T, 1997 "Feeling with the mind's eye" Neuro Report 83877 - 3881

Schlanger N, 1996 "Understanding Levallois: lithic technology and cognitive archaeology" Cambridge Archaeological Journal $6231-254$

Schroeder C E, Smiley J, Fu K G, McGinnis T, O’Connell M N, Hackett T A, 2003 “Anatomical mechanisms and functional implications of multisensory convergence in early cortical processing" International Journal of Psychophysiology 50 5-17

Shepard R N, Metzler J, 1971 "Mental rotation of three-dimensional objects" Science 171 $701-703$

Stoltz-Loike M, Bornstein M H, 1987 "The roles of imagery, language and metamemory in crossmodal transfer in children" Psychological Research 49 63-68

Streri A, Molina M, 1994 "Constraints on intermodal transfer between touch and vision in infancy", in The Development of Intersensory Perception: Comparative Perspectives Eds D J Lewkowicz, R Lickliter (Hove, Sussex: Lawrence Erlbaum Associates) pp 285-307

Vanlierde A, De Volder A, Wanet-Defalque M-C, Veraart C, 2003 "Occipito-parietal cortex activation during visuo-spatial imagery in early blind humans" NeuroImage $19698-709$

Woods A T, O'Modhrain S, Newell F N, 2004 "The effect of temporal delay and spatial differences in crossmodal object recognition" Cognitive, Affective, \& Behavioral Neuroscience $4260-269$

Zhang M, Weisser V D, Stilla R, Prather S C, Sathian K, 2004 "Multisensory cortical processing of object shape and its relation to mental imagery" Cognitive, Affective, \& Behavioral Neuroscience $4251-259$ 


\section{PERTEPTION}

VOLUME 362007

www.perceptionweb.com

Conditions of use. This article may be downloaded from the Perception website for personal research by members of subscribing organisations. Authors are entitled to distribute their own article (in printed form or by e-mail) to up to 50 people. This PDF may not be placed on any website (or other online distribution system) without permission of the publisher. 